\title{
Capsule commentary on Jones et al., "Connecting the Dots": a Qualitative Study of Home Health Nurse Perspectives on Coordinating Care for Recently-Discharged Patients
}

\author{
Jessica A. Eng, MD MS ${ }^{1,2}$

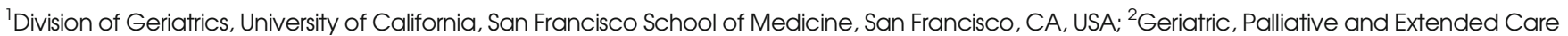 \\ Services, San Francisco VA Health Care System, San Francisco, CA, USA.
}

J Gen Intern Med 32(10): 1134

DOI: $10.1007 / \mathrm{s} 11606-017-4129-4$

() Society of General Internal Medicine 2017

$\mathrm{T}$ his qualitative study by Jones et al. ${ }^{1}$ investigates the challenges and potential solutions for the poor communication between hospitals and home health care (HHC) services. The investigators used select domains from an existing care coordination framework to conduct focus groups with over $50 \mathrm{HHC}$ services nurses and administrators from 6 agencies. The challenges and potential solutions were mapped to four previously identified domains (Accountability, Communication, Assessing Needs and Goals, and Medication Management) and one additional domain identified during thematic analysis (Safety). The authors found that efforts to improve care coordination with $\mathrm{HHC}$ services should focus on defining accountability for orders during transitions, improved communication, alignment of expectations for HHC services, a focus on reducing medication discrepancies, and increased awareness of safety issues for both patients and HHC nurses.

With increasing shared accountability for outcomes and costs, care is increasingly shifting from inpatient wards to outpatient clinics to patients' homes, and research and quality improvement efforts must follow and study these shifts in the care location. In particular, the need for greater research on transitions of care involving HHC services is clear given the high percentage of patients experiencing adverse events posthospitalization $^{2}$ and the rising number of $\mathrm{HHC}$ referrals. ${ }^{3}$ The existing literature on $\mathrm{HHC}$ services has focused on the frequency and type of adverse events ${ }^{4}$ but has not deeply explored how and why those errors occur.

For clinicians and administrators, this study points to the need to engage with HHC services directly in this new era of shared accountability. Future directions to improve transition of care involving HHC services should focus on making hospital electronic health record access and direct phone lines to accountable clinicians available to HHC agencies. With improved communication access, HHC agencies can be true partners in the care of patients during the tenuous post-discharge period.

Corresponding Author: Jessica A. Eng, MD MS; Division of GeriatricsUniversity of California, San Francisco School of Medicine, San Francisco, CA, USA (e-mail: jessica.eng@va.gov).

Compliance with Ethical Standards:

Conflict of Interest: The author declares that she does not have a conflict of interest.

\section{REFERENCES}

1. Jones CD, Jones J, Richard A, et al. "Connecting the dots": a qualitative study of home health nurse perspectives on coordinating care for recentlydischarged patients. J Gen Intern Med. doi: 10.1007/s11606-017-4104-0.

2. Forster AJ, Murff HJ, Peterson JF, Gandhi TK, Bates DW. The incidence and severity of adverse events affecting patients after discharge from the hospital. Ann Intern Med. 2003; 138: 161-167

3. Jones CD, Ginde AA, Burke RE, Wald HL, Masoudi FA, Boxer RS. Increasing home healthcare referrals upon discharge from US hospitals: 2001-2012. J Am Geriatr Soc. 2015;63(6): 1265-1266.

4. Press MJ, Gerber LM, Peng TP, Pesko MF, Feldman PH, Ouchida K, Sridharan S, Bao Y, Barron Y, Casalino LP. Postdischarge communication between home health nurses and physicians: measurement, quality, and outcomes. J Am Geriatr Soc. 2015 63(7): 1299-1305. 